\title{
Analytical Solution of the Problem of Vibration of Plates with Piezoelectric Actuators with Arbitrary Shape in Distribution Formulation
}

\author{
M. WICIAK* \\ Institute of Mathematics, Cracow University of Technology, Warszawska 24, 31-155 Cracow, Poland
}

\begin{abstract}
This paper is concerned with mathematical aspects of modelling vibration of a plate with piezoelectric actuators. Particularly, a thin Kirchhoff-Love plate with arbitrary shaped actuators (e.g. triangles, parallelograms, discs) is considered. The moments that act upon a structure and are induced by piezoelectric actuators, are described by the generalized tensor product of a distribution and distribution-valued function. Finally, the formula for the solution of the Cauchy problem in the class of absolutely continuous tempered distribution-valued functions is derived.
\end{abstract}

PACS: 43.40.At, 43.40.Vn

\section{Introduction}

Noise and vibration are persistent problems in many industrial applications. It occurs that the usage of passive methods to give the solution is inadequate in numerous cases. The possibility of active noise and vibration control has been explored for over 50 years [1]. The advantage of using different shaped, distributed actuators for active control has been demonstrated by a number of researchers e.g. [2-4]. Originated by Sobolev [5] and Schwartz [6] theory of distributions is modern mathematical tool. Its origins reach back to physical problems where to describe some phenomena by the notion of function was inadequate. It also appeared that extension of the notion of the solution of a differential equation was convenient (distributional solution, weak solution). In particular, in acoustics the Dirac distributions appear in the wave equation in the case of quasi-point sources or moving sources. Also in active noise and vibration control distributions turn out to be powerful tool.

In this paper a thin Kirchhoff-Love plate with piezoelectric actuator is considered. The actuator consists of two identical piezoceramic elements adhered symmetrically to each side of the plate. The actuator is activated by applying a voltage of opposite signs to the opposing piezoelectric patches. The activated piezoelectric actuator will induce internal moments across the piezoelectric. It has been demonstrated that a shape and the location of actuator influence its ability to excite certain modes. In the case of rectangular actuators which are bonded so that their edges are parallel to the edges of a rectangular plate, the external loads can be described by a tensor product of some distributions [1]. However, in the case of differently shaped actuators (e.g. triangles in [4]) the definition of the tensor product must be extended to the case

\footnotetext{
* e-mail: mwiciak@pk.edu.pl
}

of a product of a distribution and a distribution-valued function.

\section{Notations}

Let $\mathcal{D}\left(\mathbb{R}^{n}\right)$ be the space of test functions that consists of all smooth functions with compact supports, and $\mathcal{D}^{\prime}\left(\mathbb{R}^{n}\right)$ be the space of distribution, that is the space of all linear, continuous functionals on $\mathcal{D}\left(\mathbb{R}^{n}\right)$. The simplest example of a distribution is so-called regular distribution defined for any locally summable function $\chi: \mathbb{R}^{n} \rightarrow \mathbb{R}$ by the formula

$$
[\chi](\varphi)=\int_{\mathbb{R}^{n}} \chi(x) \varphi(x) \mathrm{d} x
$$

for $\varphi \in \mathcal{D}\left(\mathbb{R}^{n}\right)[7]$.

It may be worth reminding the reader that if $\alpha=$ $\left(\alpha_{1}, \ldots, \alpha_{n}\right) \in \mathbb{N}^{n}$ is a multi-index then $\mathrm{D}^{\alpha}$ denotes the differential operator of order $|\alpha|=\alpha_{1}+\ldots+\alpha_{n}$,

$$
\mathrm{D}^{\alpha}=\left(\frac{\partial}{\partial x_{1}}\right)^{\alpha_{1}} \ldots\left(\frac{\partial}{\partial x_{n}}\right)^{\alpha_{n}},
$$

and for any distribution $T \in \mathcal{D}^{\prime}\left(\mathbb{R}^{n}\right)$ derivative $\mathrm{D}^{\alpha} T$ is a distribution defined by the formula

$$
\mathrm{D}^{\alpha} T(\varphi)=(-1)^{|\alpha|} T\left(\mathrm{D}^{\alpha} \varphi\right)
$$

for $\varphi \in \mathcal{D}\left(\mathbb{R}^{n}\right)$. In particular,

$$
\begin{aligned}
& \frac{\mathrm{d}}{\mathrm{d} x}\left[H_{a}\right](\varphi)=-\left[H_{a}\right]\left(\varphi^{\prime}\right) \\
& \quad=-\int_{a}^{+\infty} \varphi^{\prime}(x) \mathrm{d} x=\varphi(a)=\delta_{a}(\varphi)
\end{aligned}
$$

for $\varphi \in \mathcal{D}(\mathbb{R})$, where $H_{a}$ is a Heaviside step function, $H_{a}(x)=1$ for $x \geq a$ and $H_{a}(x)=0$ for $x<a$, and $\delta_{a}$ is the Dirac distribution and for any $\varphi \in \mathcal{D}(\mathbb{R})$, $\delta_{a}(\varphi)=\varphi(a)$. Thus 


$$
\frac{\mathrm{d}}{\mathrm{d} x}\left[H_{a}\right]=\delta_{a} .
$$

Recall that for any distribution $T \in \mathcal{D}^{\prime}\left(\mathbb{R}^{n}\right)$ and $\psi \in$ $\mathcal{C}^{\infty}\left(\mathbb{R}^{n}\right)$ the product $\psi T \in \mathcal{D}^{\prime}\left(\mathbb{R}^{n}\right)$ and is defined by

$$
(\psi T)(\varphi)=T(\varphi \psi)
$$

for $\varphi \in \mathcal{D}\left(\mathbb{R}^{n}\right)$, while the tensor product of distributions $T, S \in \mathcal{D}^{\prime}(\mathbb{R})$ is a distribution $S \otimes T \in \mathcal{D}^{\prime}\left(\mathbb{R}^{2}\right)$ defined as

$$
(S \otimes T)(\varphi)=S(x \mapsto T(\varphi(x, \cdot)))
$$

for $\varphi \in \mathcal{D}\left(\mathbb{R}^{2}\right)$. In the above the distribution $S \in \mathcal{D}^{\prime}(\mathbb{R})$ is assumed to operate on test functions of variable $x$, while $T \in \mathcal{D}^{\prime}(\mathbb{R})$ is a distribution on test functions of variable $y$. It is well known that for any $\varphi \in \mathcal{D}\left(\mathbb{R}^{2}\right)$ the mappings $\mathbb{R} \ni y \mapsto \varphi(x, y) \in \mathbb{R}$ for fixed $x$, and $R \ni x \mapsto T(\varphi(x, \cdot)) \in \mathbb{R}$ are test functions of variables $y$ and $x$, respectively. It is also known that the following formulae for derivatives are true [7]:

$$
\begin{aligned}
\frac{\partial^{k}}{\partial x^{k}}(S \otimes T) & =\frac{\mathrm{d}^{k}}{\mathrm{~d} x^{k}} S \otimes T, \\
\frac{\partial^{k}}{\partial y^{k}}(S \otimes T) & =S \otimes \frac{\mathrm{d}^{k}}{\mathrm{~d} y^{k}} T .
\end{aligned}
$$

As usual let $\mathcal{S}\left(\mathbb{R}^{n}\right)$ denote the space of rapidly decreasing functions, i.e.

$$
\begin{aligned}
& \mathcal{S}\left(\mathbb{R}^{n}\right)=\left\{\varphi \in \mathcal{C}^{\infty}\left(\mathbb{R}^{n}, \mathbb{C}\right): P \cdot \mathrm{D}^{\alpha} \varphi\right. \text { is bounded } \\
& \left.\forall \alpha \in \mathbb{N}^{n} \forall P \in \mathcal{P}\left(\mathbb{R}^{n}\right)\right\},
\end{aligned}
$$

where $\mathcal{P}\left(\mathbb{R}^{n}\right)$ denotes the set of all polynomials $\mathbb{R}^{n} \rightarrow \mathbb{R}$. Of course $\mathcal{S}\left(\mathbb{R}^{n}\right)$ is a Fréchet space, and $\mathcal{D}\left(\mathbb{R}^{n}\right) \subset \mathcal{S}\left(\mathbb{R}^{n}\right)$. Recall that a distribution $T \in \mathcal{D}^{\prime}\left(\mathbb{R}^{n}\right)$ is tempered when it is continuous in topology of $\mathcal{S}\left(\mathbb{R}^{n}\right)$. This is equivalent to the fact that there is the unique extension $\bar{T}$ of $T$ to $\mathcal{S}\left(\mathbb{R}^{n}\right)[4]$. It is customary to identify $T$ with its extension $\bar{T}$. The space of tempered distribution is denoted by $\mathcal{D}_{\text {temp }}^{\prime}\left(\mathbb{R}^{n}\right)$. Finally, the Fourier transform of tempered distribution $T \in \mathcal{D}_{\text {temp }}^{\prime}\left(\mathbb{R}^{n}\right)$, denoted by $\mathcal{F}(T)$ or $\hat{T}$, is defined by

$$
\hat{T}(\varphi)=\bar{T}(\hat{\varphi})
$$

for $\varphi \in \mathcal{S}\left(\mathbb{R}^{n}\right)$, where $\hat{\varphi}(\xi)=(2 \pi)^{-\frac{n}{2}} \int_{\mathbb{R}^{n}} \mathrm{e}^{-\mathrm{i} \xi \cdot y} \varphi(y) \mathrm{d} y$. For tensor product of $T, S \in \mathcal{D}_{\text {temp }}^{\prime}(\mathbb{R})$ one obtains

$$
\mathcal{F}(S \otimes T)=\hat{S} \otimes \hat{T} .
$$

It is known that the Fourier transform is invertible in the space of tempered distributions, and

$$
\mathcal{F}^{-1}=\mathcal{F}^{3} \text {. }
$$

\section{The vibration model}

Let us consider the equation of motion

$$
D \Delta^{2} w+\mu \frac{\partial^{2}}{\partial t^{2}} w=f,
$$

where $w$, a function of time variable $t$ and spatial variables $(x, y)$, is a transverse displacement, and $\Delta$ is the
Laplacian. Moreover $f$, a function of $(t, x, y)$, is the external excitation, $\mu=\rho h_{0}, \rho$ is the material density, and $h_{0}$ is the plate thickness, $D=E h_{0}^{3} / 12\left(1-\nu^{2}\right)$ is the flexural rigidity of the plate, $\nu$ and $E$ are the Poisson ratio and Young's modulus, respectively. Additionally, consider a triangle-shaped piezoelectric actuator adhered to the plate. The actuator consists of two identical triangle-shaped piezoelectric elements of thickness $h$, bonded symmetrically on two opposite surfaces of the plate as shown in Fig. 1.

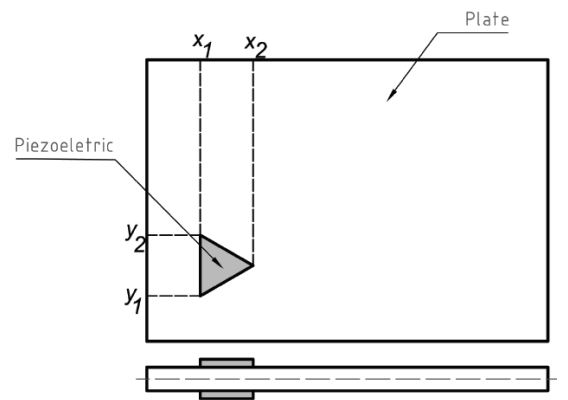

Fig. 1. Plate with triangular actuator.

The two elements are excited by opposite voltages and the actuator generates distributed reaction moments in the plate. It is assumed that the reaction moments $m_{x}$ and $m_{y}$ are equal and homogeneous over the actuator area. Let $f_{\mathrm{pe}}$, a function of $(t, x, y)$, be an additional external force caused by activating the piezoelectric element. It was demonstrated in $[1,4]$ that

$$
f_{\mathrm{pe}}=\frac{\partial^{2} m_{x}}{\partial x^{2}}+\frac{\partial^{2} m_{y}}{\partial y^{2}}
$$

and

$$
m_{x}=m_{y}=m=C_{0} \epsilon_{\mathrm{pe}} \chi,
$$

where $C_{0}$ denotes the piezoelectric strain-plate moment coupling term, $\epsilon_{\mathrm{pe}}=\left(d_{31} V\right) / h$ is the magnitude of the induced strain, $d_{31}$ piezoelectric-strain constant, $V$ is the applied voltage, and $\chi$ is a characteristic function defined on the area $\Gamma$, on which the piezoelectric actuator is bonded,

$$
\chi(x, y)=\left\{\begin{array}{l}
1 \text { if }(x, y) \in \Gamma, \\
0 \text { if }(x, y) \notin \Gamma,
\end{array}\right.
$$

while $\Gamma=\left\{(x, y): x_{1} \leq x \leq x_{2}, a_{1} x+b_{1} \leq y \leq a_{2} x+b_{2}\right\}$.

Of course the function $\chi$ is not differentiable (even not continuous) however the derivatives of $m$ can be understood in the sense of the theory of distributions. Namely, a locally summable function $\chi: \mathbb{R}^{2} \rightarrow \mathbb{R}$ defines regular distribution by the formula

$$
[\chi](\varphi)=\int_{\Gamma} \varphi(x, y) \mathrm{d} x \mathrm{~d} y
$$

for $\varphi \in \mathcal{D}\left(\mathbb{R}^{2}\right)$. It is also convenient to treat the distribution $[\chi]$ as a tensor product of distributions, $[1,4]$, one that operates on test functions of variable $x$, and the 
second one which is a distribution on test functions of variable $y$. This treatment is appropriate for the case of rectangular actuator which is bonded so that its edges are parallel to the plate edges, though with more complicated shapes of actuators one should be more careful. In particular for triangular actuator the distribution $[\chi]$ cannot be treated as a tensor product of distributions since $f_{i}(x)=a_{i} x+b_{i}$ is a function of $x$ and $\left[H_{f_{i}}\right]$ is no longer a distribution but a distribution-valued function, $\mathbb{R} \ni x \mapsto\left[H_{f_{i}(x)}\right] \in \mathcal{D}^{\prime}(\mathbb{R})$. A simple example showing that even when a distribution $S$ operates on test functions of variable $x$ and $T(x) \in \mathcal{D}^{\prime}(\mathbb{R})$ is a distribution that operates on test functions of variable $y$, the tensor product is not a distribution, can be constructed. Namely, let $T$ be a regular distribution generated by locally summable function

$$
u(x, y)=\left\{\begin{array}{l}
1 \text { if } 0 \leq x \leq 1, y \in \mathbb{R}, \\
0 \text { otherwise }
\end{array}\right.
$$

Then for any fixed $x, T(x)=[u(x, \cdot)]$ is a distribution that operates on test functions of variable $y$. But for a test function $\varphi \in \mathcal{D}\left(\mathbb{R}^{2}\right)$ such that $\operatorname{supp} \varphi \subset[0,1] \times[0,1]$, $\varphi=1$ on $\operatorname{supp} \varphi$ there is

$$
\begin{aligned}
& T(x) \varphi(x, \cdot)=[u(x, \cdot)] \varphi(x, \cdot)=\int_{\mathbb{R}} u(x, y) \varphi(x, y) \mathrm{d} y \\
& \quad= \begin{cases}\int_{\mathbb{R}} \varphi(x, y) \mathrm{d} y & \text { for } x \in[0,1], \\
0 & \text { for } x \notin[0,1],\end{cases}
\end{aligned}
$$

Thus

$$
T(x) \varphi(x, \cdot)=\left\{\begin{array}{l}
1 \text { for } x \in[0,1], \\
0 \text { for } x \notin[0,1] .
\end{array}\right.
$$

But the function $\mathbb{R} \ni x \mapsto T(x) \varphi(x, \cdot)$ is even not continuous, so it is not a test function. Consequently, there is no sense in putting $S(x \mapsto T(\varphi(x, \cdot)))$ for $S \in \mathcal{D}^{\prime}(\mathbb{R})$.

For this reason one needs to use the generalized tensor product. This notion is also called the parameter product [8]. Let a distribution-valued function $T$ : $R \ni x \mapsto T(x) \in \mathcal{D}^{\prime}(\mathbb{R})$ be of class $C^{\infty}$ i.e. a function $\mathbb{R} \ni x \mapsto T(x) \varphi \in \mathbb{R}$ is a smooth function for all $\varphi \in \mathcal{D}(\mathbb{R})$. With this assumption one can verify that the map $\mathbb{R} \ni x \mapsto T(x) \varphi(x, \cdot) \in \mathbb{R}$ is a test function for any $\varphi \in \mathcal{D}\left(\mathbb{R}^{2}\right)$. Thus it is meaningful to define the generalized tensor product of $S \in \mathcal{D}^{\prime}(\mathbb{R})$ and $T, S \otimes T$ as a distribution belonging to $\mathcal{D}^{\prime}\left(\mathbb{R}^{2}\right)$ as follows:

$$
(S \otimes T)(\varphi)=S(x \mapsto T(\varphi(x, \cdot)))
$$

for any $\varphi \in \mathcal{D}\left(\mathbb{R}^{2}\right)$. The following formulae for derivatives of $S \otimes T$ are also true

$$
\begin{aligned}
& \frac{\mathrm{d}^{k}}{\mathrm{~d} x^{k}}(S \otimes T)=\sum_{i=0}^{k}\left(\begin{array}{c}
k \\
i
\end{array}\right) \frac{\mathrm{d}^{i}}{\mathrm{~d} x^{i}} S \otimes T^{(k-i)}(\cdot), \\
& \frac{\mathrm{d}^{k}}{\mathrm{~d} y^{k}}(S \otimes T)=S \otimes \frac{\mathrm{d}^{k}}{\mathrm{~d} y^{k}}(T(\cdot))
\end{aligned}
$$

for any $k \in \mathbb{N}$, where the symbols $\partial^{k} / \partial x^{k}, \mathrm{~d}^{i} / \mathrm{d} x^{i}$, $\partial^{k} / \partial y^{k}, \mathrm{~d}^{k} / \mathrm{d} y^{k}$ denote derivatives of distributions in ac- cordance with (3), while $T^{(j)}$ means the $j$-th derivative of the map $T$, i.e.

$$
T^{(j)}(x)(\varphi)=\frac{\mathrm{d}^{j}}{\mathrm{~d} x^{j}}(T(x) \varphi)
$$

for any $\varphi \in \mathcal{D}(\mathbb{R})$.

Therefore, the internal moments across the triangular piezoelectric can be expressed as the generalized tensor product

$$
m_{x}=m_{y}=C_{0} \epsilon_{\mathrm{pe}}\left(\left[H_{x_{1}}-H_{x_{2}}\right] \otimes\left[H_{f_{1}(\cdot)}-H_{f_{2}(\cdot)}\right]\right) .
$$

Since for any $x \in \mathbb{R}$ and $\varphi \in \mathcal{D}(\mathbb{R})$ :

$$
\left[H_{f_{i}(x)}\right] \varphi=\int_{f_{i}(x)}^{\infty} \varphi(y) \mathrm{d} y, \quad i=1,2,
$$

the derivative of the map $\mathbb{R} \ni x \mapsto\left[H_{f_{i}(x)}\right] \in \mathcal{D}^{\prime}(\mathbb{R})$ can be easily found as

$$
\begin{aligned}
& {\left[H_{f_{i}(\cdot)}\right]^{\prime}(x)=-f_{i}^{\prime}(x) \delta_{f_{i}(x)}=-a_{i} \delta_{f_{i}(x)},} \\
& \quad i=1,2
\end{aligned}
$$

and

$$
\begin{aligned}
& {\left[H_{f_{i}(\cdot)}\right]^{\prime \prime}(x)=-f_{i}^{\prime \prime}(x) \delta_{f_{i}(x)}+\left(f_{i}^{\prime}(x)\right)^{2} \delta_{f_{i}(x)}^{\prime}} \\
& \quad=a_{i}^{2} \delta_{f_{i}(x)}^{\prime}, \quad i=1,2 .
\end{aligned}
$$

Thus from (25), (22) one obtains

$$
\begin{aligned}
& \frac{\partial^{2} m}{\partial x^{2}}=C_{0} \epsilon_{\mathrm{pe}}\left(\left[H_{x_{1}}-H_{x_{2}}\right] \otimes\left(a_{1}^{2} \delta_{f_{1}(\cdot)}^{\prime}-a_{2}^{2} \delta_{f_{2}(\cdot)}^{\prime}\right)\right. \\
& \quad+2\left(\delta_{x_{1}}-\delta_{x_{2}}\right) \otimes\left(a_{2} \delta_{f_{2}(\cdot)}-a_{1} \delta_{f_{1}(\cdot)}\right) \\
& \left.\quad+\left(\delta_{x_{1}}^{\prime}-\delta_{x_{2}}^{\prime}\right) \otimes\left[H_{f_{1}(\cdot)}-H_{f_{2}(\cdot)}\right]\right) .
\end{aligned}
$$

Similarly, in accordance with (25), (23)

$$
\frac{\partial^{2} m}{\partial y^{2}}=C_{0} \epsilon_{\mathrm{pe}}\left(\left[H_{x_{1}}-H_{x_{2}}\right] \otimes\left(\delta_{f_{1}(\cdot)}^{\prime}-\delta_{f_{2}(\cdot)}^{\prime}\right)\right) .
$$

Consequently,

$$
\begin{aligned}
& f_{\mathrm{pe}}(t)=C_{0} \epsilon_{\mathrm{pe}}\left(\left(\delta_{x_{1}}^{\prime}-\delta_{x_{2}}^{\prime}\right) \otimes\left[H_{f_{1}(\cdot)}-H_{f_{2}(\cdot)}\right]\right. \\
& \quad+2\left(\delta_{x_{1}}-\delta_{x_{2}}\right) \otimes\left(a_{2} \delta_{f_{2}(\cdot)}-a_{1} \delta_{f_{1}(\cdot)}\right) \\
& \left.\quad+\left[H_{x_{1}}-H_{x_{2}}\right] \otimes\left(\left(a_{1}^{2}+1\right) \delta_{f_{1}(\cdot)}^{\prime}-\left(a_{2}^{2}+1\right) \delta_{f_{2}(\cdot)}^{\prime}\right)\right) .
\end{aligned}
$$

Finally, the equation of motion for a plate with triangular piezoelectric actuator is obtained as

$$
D \Delta^{2} w(t)+\mu \frac{\mathrm{d}^{2}}{\mathrm{~d} t^{2}} w(t)=f(t)+f_{\mathrm{pe}}(t)
$$

with $f_{\text {pe }}$ given by $(31)$.

It should be noted that unknown function $w$ is now treated as an evolution in time of a two-dimensional distribution, $w:[0,+\infty) \ni t \mapsto w(t) \in \mathcal{D}^{\prime}\left(\mathbb{R}^{2}\right)$. Consequently, the second derivative with the respect to $t$ is now understood as a derivative of a function of one variable $t$ [9]. Similarly $f, f_{\mathrm{pe}}:[0,+\infty) \rightarrow \mathcal{D}^{\prime}\left(\mathbb{R}^{2}\right)$ are functions of $t$.

Observe that this method can be useful for actuators 


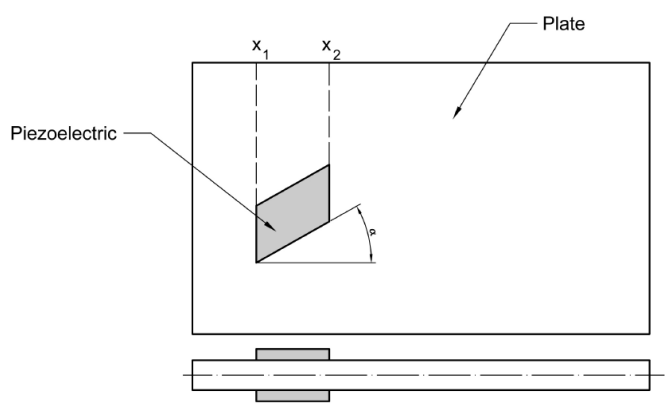

Fig. 2. Plate with parallelogram-shaped actuator.

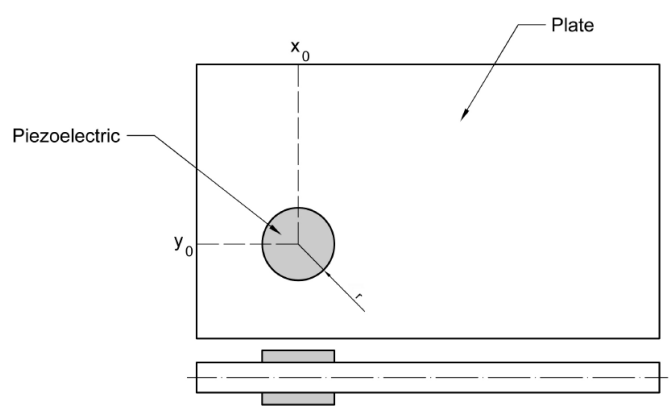

Fig. 3. Plate with disc-shaped actuator.

of more complicated shape. For example consider parallelogram and disc-shaped actuators as shown in Fig. 2 and Fig. 3. In both cases the reaction moments can be presented as

$$
m_{x}=m_{y}=m=C_{0} \epsilon_{\mathrm{pe}} \chi,
$$

where $\chi$ is a characteristic function of $\Gamma$, and

$$
\Gamma=\left\{(x, y): x_{1} \leq x \leq x_{2}, a x+b_{1} \leq y \leq a x+b_{2}\right\}
$$

( $a=\tan \alpha$ ) for parallelogram-shaped actuator, and

$$
\begin{aligned}
& \Gamma=\left\{(x, y): x_{1}=x_{0}-r \leq x \leq x_{0}+r=x_{2},\right. \\
& \left.y_{0}-\sqrt{r^{2}-\left(x-x_{0}\right)^{2}} \leq y \leq y_{0}+\sqrt{r^{2}-\left(x-x_{0}\right)^{2}}\right\}
\end{aligned}
$$

for disc-shaped actuator. Consequently

$$
m_{x}=m_{y}=C_{0} \epsilon_{\mathrm{pe}}\left(\left[H_{x_{1}}-H_{x_{2}}\right] \otimes\left[H_{f_{1}(\cdot)}-H_{f_{2}(\cdot)}\right]\right),
$$

where $f_{i}(x)=a x+b_{i}(i=1,2)$ in the case of parallelogram-shaped actuator, and $f_{1,2}(x)=y_{0} \mp$ $\sqrt{r^{2}-\left(x-x_{0}\right)^{2}}$ in the case of disc-shaped actuator. Hence in accordance with $(22,23)$ one obtains

$$
\begin{aligned}
& f_{\mathrm{pe}}(t)=C_{0} \epsilon_{\mathrm{pe}}\left(\left(\delta_{x_{1}}^{\prime}-\delta_{x_{2}}^{\prime}\right) \otimes\left[H_{f_{1}(\cdot)}-H_{f_{2}(\cdot)}\right]\right. \\
& \quad+2 a\left(\delta_{x_{1}}-\delta_{x_{2}}\right) \otimes\left(\delta_{f_{2}(\cdot)}-\delta_{f_{1}(\cdot)}\right) \\
& \left.\quad+\left(a^{2}+1\right)\left[H_{x_{1}}-H_{x_{2}}\right] \otimes\left(\delta_{f_{1}(\cdot)}^{\prime}-\delta_{f_{2}(\cdot)}^{\prime}\right)\right)
\end{aligned}
$$

for parallelogram-shaped actuator. Similarly the formula for the force caused by disc-shaped actuator is derived.
Namely

$$
\begin{aligned}
& {\left[H_{f_{i}(\cdot)}\right]^{\prime}(x)=\mp \frac{x-x_{0}}{\sqrt{r^{2}-\left(x-x_{0}\right)^{2}}} \delta_{f_{i}(x)},} \\
& \quad i=1,2
\end{aligned}
$$

and

$$
\begin{aligned}
& {\left[H_{f_{1,2}(\cdot)}\right]^{\prime \prime}(x)=\frac{1}{r^{2}-\left(x-x_{0}\right)^{2}}} \\
& \quad \times\left(\mp \frac{r^{2}}{\sqrt{r^{2}-\left(x-x_{0}\right)^{2}}} \delta_{f_{1,2}(x)}+\left(x-x_{0}\right)^{2} \delta_{f_{1,2}(x)}^{\prime}\right) .
\end{aligned}
$$

Finally

$$
\begin{aligned}
& f_{\mathrm{pe}}(t)=C_{0} \epsilon_{\mathrm{pe}}\left(\left(\delta_{x_{1}}^{\prime}-\delta_{x_{2}}^{\prime}\right) \otimes\left[H_{f_{1}(\cdot)}-H_{f_{2}(\cdot)}\right]\right. \\
& -2\left(\delta_{x_{1}}-\delta_{x_{2}}\right) \otimes \frac{x-x_{0}}{\sqrt{r^{2}-\left(x-x_{0}\right)^{2}}}\left(\delta_{f_{2}(\cdot)}+\delta_{f_{1}(\cdot)}\right) \\
& -\left[H_{x_{1}}-H_{x_{2}}\right] \otimes \frac{r^{2}}{\left[r^{2}-\left(x-x_{0}\right)^{2}\right]^{\frac{3}{2}}}\left(\delta_{f_{2}(\cdot)}+\delta_{f_{1}(\cdot)}\right) \\
& +\left[H_{x_{1}}-H_{x_{2}}\right] \otimes \frac{r^{2}}{\left[r^{2}-\left(x-x_{0}\right)^{2}\right]^{\frac{3}{2}}} \\
& \left.\quad \times\left(\delta_{f_{1}(\cdot)}^{\prime}-\delta_{f_{2}(\cdot)}^{\prime}\right)\right) .
\end{aligned}
$$

It may be worth to note that the presented method is also applicable to the case of a system of piezoelectric actuators. In this case the additional term $f_{\text {pe }}$ on the right-hand side of (32) can be represented as a sum $f_{\mathrm{pe}}=\sum_{i} f_{\mathrm{pe}}^{i}$, where $f_{\mathrm{pe}}^{i}$ is the force caused by the $i$-th piezoactuator.

The solution of Eq. (32) with appropriate boundary conditions can be found using finite element method (FEM). At the same time an analytical formula for the solution is possible to gain. Section 4 is devoted to a method of getting this formula. The assumption of the application of the method is that the right-hand side of (32) is a tempered distribution.

\section{The analytical solution to vibration problem}

An analytical solution to the Cauchy problem related to Eq. (32) can be obtained with the use of the Fourier transformation. The assumption of the application of the method is that the right-hand side of (32) is a tempered distribution. Assume that $S \in \mathcal{D}_{\text {temp }}^{\prime}(\mathbb{R})$ and a distribution-valued function $T: \mathbb{R} \ni x \mapsto T(x) \in$ $\mathcal{D}_{\text {temp }}^{\prime}(\mathbb{R})$ is of class $\mathcal{C}^{\infty}$. If additionally the map $T$ is polynomially bounded together with all its derivatives, i.e. the function $\mathbb{R} \ni x \mapsto T(x) \varphi$ is polynomially bounded together with all its derivatives for all $\varphi \in \mathcal{S}(\mathbb{R})$, it can be proved that the generalized tensor product $S \otimes T$ is a tempered distribution, and

$$
(S \otimes T) \varphi=S(x \mapsto T(x) \varphi(x, \cdot))
$$

for any $\varphi \in \mathcal{S}\left(\mathbb{R}^{2}\right)$. In the case of $f_{\text {pe }}$ given by (31) it is easy to observe that for any $\varphi \in \mathcal{S}(\mathbb{R})$ the functions 


$$
\begin{aligned}
& x \mapsto\left[H_{f_{i}(x)}\right] \varphi=\int_{f_{i}(x)}^{+\infty} \varphi(y) \mathrm{d} y, \\
& x \mapsto \delta_{f_{i}(x)} \varphi=\varphi\left(f_{i}(x)\right), \\
& x \mapsto \delta_{f_{i}(x)}^{\prime} \varphi=-\frac{\mathrm{d}}{\mathrm{d} x} \varphi\left(f_{i}(x)\right)
\end{aligned}
$$

are polynomially bounded together with all its derivatives, and so

$$
\begin{aligned}
x & \mapsto\left[H_{f_{i}(x)}\right] \varphi(x, \cdot)=\int_{f_{i}(x)}^{+\infty} \varphi(y) \mathrm{d} y, \\
x & \mapsto \delta_{f_{i}(x)} \varphi(x, \cdot)=\varphi\left(x, f_{i}(x)\right), \\
x & \mapsto \delta_{f_{i}(x)}^{\prime} \varphi(x, \cdot)=-\frac{\partial}{\partial x} \varphi\left(x, f_{i}(x)\right),
\end{aligned}
$$

are rapidly decreasing for any $\varphi \in \mathcal{S}\left(\mathbb{R}^{2}\right)$. Since distributions $\delta_{x_{i}}, \delta_{x_{i}}^{\prime},\left[H_{x_{i}}\right]$ are tempered, generalized tensor products

$$
\left[H_{x_{i}}\right] \otimes \delta_{f\left(x_{i}\right)}^{\prime}, \quad \delta_{x_{i}} \otimes \delta_{f\left(x_{i}\right)}, \quad \delta_{x_{i}}^{\prime} \otimes\left[H_{f_{i}(x)}\right]
$$

are also tempered. Similarly, one can show that distributions in (37) and (40) are tempered.

Now turn to the Cauchy problem associated to Eq. (32),

$$
\left\{\begin{array}{l}
D \Delta^{2} w(t)+\mu \frac{\mathrm{d}^{2}}{\mathrm{~d} t^{2}} w(t)=f(t)+f_{\mathrm{pe}}(t)+f_{b}(t), \\
w(0)=w_{0}, \\
w^{\prime}(0)=w_{1},
\end{array}\right.
$$

with given initial conditions: $w_{0}, w_{1} \in \mathcal{D}_{\text {temp }}^{\prime}\left(\mathbb{R}^{2}\right)$, and locally summable external forces $f, f_{\mathrm{pe}}:[0,+\infty) \rightarrow$ $\mathcal{D}_{\text {temp }}^{\prime}\left(\mathbb{R}^{2}\right)$. In the usual manner the boundaries conditions can be also included in (49) in terms of additional element $f_{b}:[0,+\infty) \rightarrow \mathcal{D}_{\text {temp }}^{\prime}\left(\mathbb{R}^{2}\right)$ on the right hand-side of the equation. Since $f_{\mathrm{pe}}:[0,+\infty) \rightarrow \mathcal{D}_{\text {temp }}^{\prime}\left(\mathbb{R}^{2}\right)$ has values in the space of tempered distributions, it is meaningful to search a solution $w$ in the class of absolutely continuous functions, $w:[0,+\infty) \rightarrow \mathcal{D}_{\text {temp }}^{\prime}\left(\mathbb{R}^{2}\right)$, in the sense of [9].

\section{Setting}

$$
u=\left(u_{1}, u_{2}\right): t \mapsto\left(w(t), w^{\prime}(t)\right) \in \mathcal{D}_{\text {temp }}^{\prime}\left(\mathbb{R}^{2}\right)
$$

the problem (49) can be rewritten as a first order Cauchy problem

$$
\left\{\begin{array}{l}
\mu \frac{\mathrm{d}}{\mathrm{d} t} u(t)=\sum_{|\alpha| \leq 4} A_{\alpha} \mathrm{D}^{\alpha} u(t)+F(t) \text { for a.e. } t \geq 0 \\
u(0)=u_{0}
\end{array}\right.
$$

where $\mathrm{D}^{\alpha}$ is the differential operator in the sense of (3) of order $|\alpha|=\alpha_{1}+\alpha_{2}$, for multi-index $\alpha \in \mathbb{N}^{2}, F(t)=$ $\left(0, f(t)+f_{\mathrm{pe}}(t)+f_{b}(t)\right), u_{0}=\left(w_{0}, w_{1}\right) \in \mathcal{D}_{\text {temp }}^{\prime}\left(\mathbb{R}^{2}\right)$, and

$$
A_{(0,0)}=\left(\begin{array}{ll}
0 & 1 \\
0 & 0
\end{array}\right), \quad A_{(4,0)}=A_{(0,4)}=-\frac{D}{\mu}\left(\begin{array}{ll}
0 & 0 \\
0 & 1
\end{array}\right)
$$

$$
A_{(2,2)}=-2 \frac{D}{\mu}\left(\begin{array}{ll}
0 & 0 \\
0 & 1
\end{array}\right) .
$$

$A_{\alpha}=0$ for any other multi-index $|\alpha| \leq 4$. The existence and the uniqueness of a solution of (49) follows from the theorem 46 [9]. Actually, the eigenvalues of the matrix

$$
\sum_{|\alpha| \leq 4}(\mathrm{i} \xi)^{\alpha} A_{\alpha}=\left(\begin{array}{ll}
0 & 1 \\
0 & 0
\end{array}\right)-\frac{D}{\mu}|\xi|^{4}\left(\begin{array}{ll}
0 & 0 \\
0 & 1
\end{array}\right),
$$

where $|\xi|^{2}=\xi_{1}^{2}+\xi^{2}$ are

$$
-\mathrm{i} \sqrt{\frac{D}{\mu}}|\xi|^{2}, \quad \mathrm{i} \sqrt{\frac{D}{\mu}}|\xi|^{2},
$$

thus there is a unique solution of the problem (51) given by the formula

$$
\begin{aligned}
& u(t)=\mathcal{F}^{-1}\left(\mathrm{e}^{t \sum_{|\alpha| \leq 4}(\mathrm{i} \xi)^{\alpha} A_{\alpha}} \widehat{u_{0}}\right) \\
& +\int_{0}^{t} \mathcal{F}^{-1}\left(\mathrm{e}^{(t-s) \sum_{|\alpha| \leq 4}(\mathrm{i} \xi)^{\alpha} A_{\alpha}} \widehat{F(s)}\right) \mathrm{d} s,
\end{aligned}
$$

where $\mathcal{F}^{-1}$ is the inverse Fourier transform (12) of the tempered distribution.

Finally, one obtains the formula for the solution of (49)

$$
\begin{aligned}
& w(t)=\mathcal{F}^{-1}\left(\cos \left(\sqrt{\frac{D}{\mu}}|\xi|^{2} t\right) \widehat{w}_{0}\right) \\
& +\mathcal{F}^{-1}\left(\frac{1}{\sqrt{\frac{D}{\mu}}|\xi|^{2}} \sin \left(\sqrt{\frac{D}{\mu}}|\xi|^{2} t\right) \widehat{w_{1}}\right) \\
& +\int_{0}^{t} \mathcal{F}^{-1}\left(\frac{1}{\sqrt{\frac{D}{\mu}}|\xi|^{2}} \sin \left(\sqrt{\frac{D}{\mu}}|\xi|^{2}(t-s)\right)\right. \\
& \left.\quad \times\left[\widehat{f(s)}+\widehat{f_{\mathrm{pe}}(s)}+\widehat{f_{b}(s)}\right]\right) \mathrm{d} s .
\end{aligned}
$$

\section{Conclusions}

Some mathematical problems in modelling vibration of a plate with piezoelectric actuators of arbitrary shape were discussed. The internal moments across the actuator were described in terms of generalized tensor product of a distribution and a distribution-valued function. Finally the formula for the unique solution in the class of absolutely continuous distribution-valued functions was demonstrated. Presented method is also applicable to the case of a system of piezoelectric actuators.

\section{Acknowledgments}

This study is a part of the research project N N504 078038 supported by the Ministry of Science and Higher Education, Poland. 


\section{References}

[1] E.K. Dimitriadis, C.R. Fuller, C.A. Rogers, J. Vib. Acoust. 113, 100 (1991).

[2] P. Gardonio, S.J. Elliott, J. Acoust. Soc. Am. 117, 2046 (2005).

[3] E.M. Sekouri, Y.R. Hu, A.D. Ngo, Mechatronics 14, 1007 (2004).

[4] J.M. Sullivan, J.E. Hubbard, Jr., S.E. Burke, J. Acoust. Soc. Am. 99, 2965 (1996).
[5] L.S. Sobolev, Mat. Sb. 1, 39 (1936) (in French).

[6] L. Schwartz, Théorie des distributions, Hermann, Paris 1951.

[7] W. Rudin, Functional Analysis, McGraw-Hill, New York 1973.

[8] H.G. Embacher, G. Grubl, M. Oberguggenberger, Z. Analysis Anwendungen 11, 437 (1992).

[9] M. Wiciak, Univ. Iagel. Acta Math. 42, 31 (2004). 